\title{
Plasma vascular endothelial growth factor, soluble VEGF receptor FLT-1, and von Willebrand factor in glaucoma
}

\author{
P L Lip, D C Felmeden, A D Blann, N Matheou, S Thakur, I A Cunliffe, G Y H Lip
}

Br J Ophthalmol 2002;86: 1299-1302

See end of article for authors' affiliations

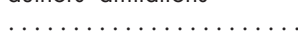

Correspondence to: Professor G Y H Lip, University Department of Medicine, City Hospital, Birmingham B18 7QH,

UK; G.Y.H.LIP@bham.ac.uk

Accepted for publication 15 May 2002

\begin{abstract}
Aim: To investigate plasma indices of vascular permeability (vascular endothelial growth factor, VEGF - also an index of angiogenesis, as well as the soluble receptor for VEGF, sFlt-1) and endothelial damage/dysfunction (von Willebrand factor, vWf) in glaucoma.

Methods: Citrated plasma was assayed for VEGF, sFlt- 1 , and vWf (all ELISA) in a cross sectional study of 50 patients (20 male; mean age 63.9 years, SD 10.5) with glaucoma: 26 had normal tension glaucoma (NTG) and 24 had primary open angle glaucoma (POAG), who were compared with 26 healthy controls (mean age 73.4 years, SD 9.2).

Results: Median (interquartile range, IQR) levels of VEGF were significantly elevated in patients with NTG and POAG compared to healthy controls (Kruskal-Wallis test, $p<0.001$ ). Similarly, mean (SD) vWF levels were abnormal in NTG and POAG compared to healthy controls (one way ANOVA, $p<0.001)$. Median levels of sFlt-1 were significantly lower in patients with NTG and POAG, when compared to healthy controls (Kruskal-Wallis test, $p<0.001 ; p<0.05$ with Tukey's post hoc test for controls $v$ POAG). There were no significant differences in VEGF, sFlt-1 or vWf levels between the NTG and POAG groups (Tukey's test, all p=NS). In both NTG and POAG groups, there was a significant correlation between VEGF and sFlt-1 (Spearman, NTG: $r=0.6517, p=0.001$; POAG: $r=0.6017$, $p=0.008)$. There were no significant correlations between VEGF and sFlt- 1 , or with vWf among the controls.

Conclusions: The pathogenesis of optic nerve damage in both NTG and POAG may be associated with abnormal vascular permeability and endothelial damage/dysfunction, as indicated by abnormal plasma VEGF and vWf levels in these patients.
\end{abstract}

VEGF interacts with endothelial cells via two high affinity membrane spanning receptors, Flt-1 and KDR. The role of receptors in embryonic vasculogenesis and adult angiogenesis and its association with several diseases has been clearly established, ${ }^{19}$ and the presence of this receptor has also been identified on retinal endothelial cells and pericytes. A soluble form of Flt-1 (sFlt-1) has been identified in conditioned culture media of human umbilical vein endothelial cells, although the pathophysiological significance of this is uncertain. ${ }^{20}$

We hypothesised that abnormal VEGF levels in NTG may reflect abnormalities in the retinal vasculature, as evident (for example) in diabetic retinopathy. ${ }^{17}{ }^{18}$ We investigated this further by undertaking a cross sectional pilot study of plasma indices of vascular permeability/angiogenesis (VEGF, vascular endothelial growth factor), soluble VEGF receptor Flt-1 (sFlt-1) and endothelial damage/dysfunction (vWf) in patients with NTG, which will be compared to levels in patients with POAG and healthy controls.

\section{PATIENTS AND METHODS}

We undertook a cross sectional study of patients with glaucoma and healthy controls. Patients with an established diagnosis of NTG and POAG were recruited from specialist glaucoma clinics at the Birmingham and Midland Eye Centre and Birmingham Heartlands Hospital. NTG was diagnosed using the following criteria: intraocular pressures $<21 \mathrm{~mm} \mathrm{Hg}$ on phasing, open angles on gonioscopy, pathological disc cupping, visual field defect and a normal intracranial neuroimaging and normal blood tests to exclude anaemic and syphilitic optic neuropathy. POAG was diagnosed using the following criteria: pathological cupping of the optic nerve head associated with raised intraocular pressure and characteristic 
Table 1 VEGF, soluble VEGF receptor Flt-1, and vWf in glaucoma compared to controls

\begin{tabular}{|c|c|c|c|c|}
\hline & NTG & POAG & Controls & $\mathrm{p}$ Value \\
\hline Number & 26 & 24 & 26 & \\
\hline Age (years) & $72.9(10.2)$ & $75.1(11.0)$ & $73.4(9.2)$ & 0.744 \\
\hline Median duration of known glaucoma (years) & 1.5 & 3.5 & $\ldots$ & 0.04 (Mann-Whitney test) \\
\hline \multicolumn{5}{|l|}{ Past medical history (n) } \\
\hline Hypertension (treated) & 7 & 13 & $\cdots$ & \\
\hline Ischaemic heart disease & 5 & 5 & & \\
\hline Cerebrovascular disease & 2 & 3 & & \\
\hline Body mass index $\left(\mathrm{kg} / \mathrm{m}^{2}\right)$ & $25.5(3.8)$ & $24.7(4.5)$ & $\cdots$ & 0.53 (t-test) \\
\hline Current smokers (n) & 3 & 1 & 3 & \\
\hline Receiving treatment for glaucoma (n) & 14 & 20 & $\because i$ 准 & \\
\hline Systolic BP (mm Hg) & $148.2(21.6)$ & $150.5(24.7)$ & $144.3(28.0)$ & 0.694 \\
\hline Diastolic BP $(\mathrm{mm} \mathrm{Hg})$ & $84(7.3)$ & $86.7(13.8)$ & $79.8(7.4)$ & 0.051 \\
\hline$\%$ with blood pressure $>160 / 90 \mathrm{~mm} \mathrm{Hg}$ & $31 \%$ & $41 \%$ & $\ldots$ & \\
\hline VEGF $(\mathrm{pg} / \mathrm{ml})$ & $225 *(110-500)$ & $150 *(118-235)$ & $83(13-125)$ & $<0.001$ \\
\hline sFlt-1 (ng/ml) & $17(6-60)$ & $6 *(2-19)$ & $28(18-39)$ & 0.003 \\
\hline von Willebrand factor (IU/dl) & $180(53)^{*}$ & $189(80)^{*}$ & 91 (19) & $<0.001$ \\
\hline
\end{tabular}

Values are mean (SD), except for VEGF and sFlt-1, expressed as median (IQR)

NTG = normal tension glaucoma; $P O A G=$ primary open angle glaucoma; BP = blood pressure; VEGF = vascular endothelial growth factor; $5 F$ lt- $1=$ soluble VEGF receptor Flt-1.

${ }^{*} \mathrm{p}<0.05 v$ controls, by Tukey's post hoc test (after logarithmic transformation for VEGF and sFlt-1).

visual field loss. Following informed consent, the patients attended the glaucoma research clinic for completion of a standard proforma including demography and measurement of blood pressure using a conventional mercury sphygmomanometer. Exclusion criteria were patients with confirmed diabetes, underlying neoplasm, or connective tissue, inflammatory or infective disorders, as well as those taking antithrombotic therapy or hormone replacement therapy. A 20 $\mathrm{ml}$ blood samples was taken from an antecubital vein with minimal haemostasis. Samples are anticoagulated and centrifuged at $3000 \mathrm{rpm}$ and $4^{\circ} \mathrm{C}$ for 20 minutes. The platelet free plasma was immediately separated and frozen at $-80^{\circ} \mathrm{C}$.

Blood indices in patients with glaucoma were compared with healthy controls, which comprised staff members and patients admitted for minor surgical procedures (such as hernia repair, excision of sebaceous cysts, etc). The subjects were non-smokers with no clinical evidence of vascular, metabolic, neoplastic, or inflammatory disease, by careful history, examination, and routine laboratory tests. These subjects were normotensive and in sinus rhythm, and were not taking aspirin, warfarin, lipid lowering or antihypertensive drugs, nonsteroidal anti-inflammatory drugs, or antibiotics.

\section{Laboratory}

Citrated plasma VEGF and sFlt-1 were measured by ELISAs using commercially available reagents and recombinant standards (R\&D Systems, Abingdon, UK), as previously described. ${ }^{28}$ The VEGF assay has a minimum sensitivity of 10 $\mathrm{pg} / \mathrm{ml}$, with an intra-assay coefficient of variation (CV) of $4.9 \%$ $(\mathrm{n}=18)$ and an interassay $\mathrm{CV}$ of $6.4 \%(\mathrm{n}=40)$ at $1.6 \mathrm{pg} / \mathrm{ml}$. The sFlt- 1 assay has a minimum sensitivity of $50 \mathrm{pg} / \mathrm{ml}$, an intraassay CV of $3.7 \%(n=12)$ and an interassay CV of $8.8 \%(n=22)$ at $10 \mathrm{ng} / \mathrm{ml}$. Levels of vWf was measured using an established ELISA technique using commercial polyclonal antisera (Dako, High Wycombe, UK). Intra-assay and interassay coefficients of variation for assays are $<5 \%$ and $<10 \%$ respectively.

\section{Power calculation}

We hypothesised that there would be a graded increase in levels of vWf among healthy controls, POAG, and NTG. To be precise, that the level POAG would be two thirds of a standard deviation higher than in the healthy controls, and that levels would be a further two thirds of one standard deviation higher in the patients with NTG. In order to achieve this we needed good data from 18 subjects per group to provide power of 0.80 for a difference of $\mathrm{p}<0.01$ by analysis of variance. However, as we intended to measure two other variables (VEGF, sFlt-1) we increased our numbers accordingly. We proposed to recruit 25 patients in each of the three groups.

\section{Statistical methods}

Data are expressed as mean (SD) or (for VEGF and sFlt-1) as median (IQR, interquartile range). Comparisons between cases and controls performed using the $\chi^{2}$ test, unpaired $t$ test or Mann-Whitney test, one way ANOVA or Kruskal-Wallis test as appropriate. Intergroup comparisons were undertaken using Tukey's posthoc test, after log transformation for non-parametrically distributed variables. The interactions between high blood pressure $(>160 / 90 \mathrm{~mm} \mathrm{Hg})$ and subgroups of glaucoma (NTG, POAG) were explored using ANOVA for unbalanced data (general linear model analysis). Correlations between various markers were performed using Spearman rank correlation. Relations between clinical variables (age, sex, blood pressure, hypertension, vascular disease (that is, ischaemic heart disease or cerebrovascular disease, by conventional clinical definitions), etc) and various markers were explored using stepwise multiple regression analysis. A probability of $\mathrm{p}<0.05$ was considered as statistically significant.

\section{RESULTS}

We studied 50 patients (20 male; mean age 63.9 years (SD 10.5)) with glaucoma: 26 had NTG and 24 had POAG, who were compared with 26 healthy controls (mean age 73.4 years, (9.2)).

\section{Cross sectional analyses}

Median levels of VEGF were significantly elevated in patients with NTG and intermediate in POAG, when compared to healthy controls (Kruskal-Wallis test, $\mathrm{p}<0.001$; $\mathrm{p}<0.05$ with Tukey's posthoc test for controls $v$ NTG and POAG). Similarly, mean vWF levels were elevated in NTG and POAG, compared to controls ( $180 \mathrm{IU} / \mathrm{dl}, 189 \mathrm{IU} / \mathrm{dl}$. and $91 \mathrm{IU} / \mathrm{dl}$ respectively; one way ANOVA, $\mathrm{p}<0.001 ; \mathrm{p}<0.05$ with Tukey's posthoc test for controls $v$ NTG and POAG) (Table 1 ).

Median levels of sFlt-1 were significantly lower in patients with NTG and POAG, when compared to healthy controls (Kruskal-Wallis test, $\mathrm{p}<0.001 ; \mathrm{p}<0.05$ with Tukey's posthoc test for controls $v$ POAG). There were no significant differences in VEGF, sFlt-1, and vWf levels between the NTG and POAG groups (Tukey's test, $\mathrm{p}=\mathrm{NS}$ )

\section{Subgroup analyses}

Of the whole cohort, 18 patients $(36 \%)$ had blood pressures of $>$ 160/90 mm Hg. When these patients were compared with 
Table 2 Spearman rank correlations among VEGF, sFlt-1, and vWf in patients with glaucoma compared to controls

\begin{tabular}{lllll}
\hline & Versus & NTG & POAG & $\begin{array}{l}\text { Healthy } \\
\text { controls }\end{array}$ \\
\hline VEGF & sflt-1 & 0.6517 & 0.6017 & 0.3009 \\
& & $p=0.001$ & $p=0.008$ & 0.135 \\
& vWf & -0.1096 & 0.1206 & -0.0133 \\
& & $p=0.610$ & $p=0.633$ & $p=0.949$ \\
vWf & sFlt-1 & -0.301 & -0.1586 & 0.26 \\
& & $p=0.144$ & $p=0.492$ & $p=0.20$ \\
\hline
\end{tabular}

normotensive patients, there were no significant differences in median VEGF (Mann-Whitney test, 257 v $150 \mathrm{pg} / \mathrm{ml}, \mathrm{p}=0.09$ ) and sFlt- $1(16 v 15 \mathrm{ng} / \mathrm{ml}, \mathrm{p}=0.6 \mathrm{l})$, or mean $\mathrm{vWf}$ (unpaired $t$ test, $161 v 179 \mathrm{IU} / \mathrm{dl}, \mathrm{p}=0.356)$ levels. When the interactions between high blood pressure (>160/90 $\mathrm{mm} \mathrm{Hg}$ ) and subgroups of glaucoma (NTG, POAG) were explored using ANOVA for unbalanced data (general linear model analysis), there were no significant effects on median VEGF and sFlt-1, or mean vWf levels (all $p=N S$, data not shown). Being on treatment for glaucoma also did not influence levels of the research indices, nor did a previous history of vascular disease (all $\mathrm{p}=\mathrm{NS}$, data not shown)

\section{Correlations and multivariate analysis}

In both NTG and POAG groups, there was a significant correlation between VEGF and sFlt-1 (Spearman, NTG: $r=0.6517$, $\mathrm{p}=0.001$; POAG: $r=0.6017, \mathrm{p}=0.008$ ) (Table 2). There were no significant correlations between VEGF and sFlt-1 with vWf among the controls. There were no significant correlations among VEGF, sFlt-1, or vWF with age, sex, systolic blood pressure, or diastolic blood pressure in the NTG, POAG, and control groups (Spearman, all $\mathrm{p}=\mathrm{NS}$ ).

Using stepwise multiple regression analyses, there were no significant associations between VEGF or sFlt- 1 levels and sex, blood pressure levels or the proportion who were hypertensive (systolic blood pressure $>160 \mathrm{~mm} \mathrm{Hg}$ plus or minus diastolic blood pressure $>90 \mathrm{~mm} \mathrm{Hg}$ ) or had associated vascular disease (ischaemic heart disease or cerebrovascular disease) (data not shown). Age was the only significant determinant of vWf levels $\left(R^{2}=12.8, p<0.05\right)$

\section{DISCUSSION}

This study is limited by its cross sectional nature and the relatively small numbers of patients studied, but is adequately powered $(>80 \%)$ to show differences between cases and controls. While plasma levels of various indices have been related to intraocular pathology, ${ }^{21-23}$ there is also possibility that the abnormal plasma VEGF, sFlt-1, and von Willebrand factor do not necessarily imply its involvement in glaucoma; for example, these factors may also be related to underlying (manifest or silent) vascular disease-although no significant relation was found on subgroup analyses or stepwise multiple regression analysis, in the present study. Importantly, this study cannot determine causality, as whether the changes precede or are a consequence of glaucoma is uncertain. This can only be answered in a prospective longitudinal study of a large cohort of patients at risk of developing glaucoma, to ascertain whether various parameters at baseline are predictive. Although being treated for glaucoma made no difference to the levels of measured indices, we have not attempted to distinguish the effects of individual treatments, as the precise effects of treatment for glaucoma on these indices would need to be determined in a prospective study in untreated patients.
Nevertheless, the present study delivers an association among VEGF, sFlt-1, and vWf, and glaucoma, and is in keeping with the hypothesis that NTG development may have a relation to "vascular factors."

Abnormal blood viscosity and peripheral vascular endothelial dysfunction have previously been related to eye disease. For example, abnormal haemorheology and endothelial dysfunction have been associated with microvascular disturbance in patients with diabetes mellitus complicated by proliferative retinopathy ${ }^{24}$ and in retinal vascular occlusions. ${ }^{21} 25$ In a similar way, increased abnormal haemorheology and endothelial damage/dysfunction might have a role in the pathogenesis of optic nerve damage in NTG. vWf is an established marker of endothelial cell damage, which is affected in many vascular disorders and has prognostic implications. ${ }^{26}$ Increased levels of $\mathrm{vWf}$ are secreted by proliferating endothelial cells relative to quiescent cells in vitro, and it has been suggested that raised levels in vivo, in some variants of inflammatory vascular disease may reflect increased production from new, proliferating microvessels in the artery wall. ${ }^{27}{ }^{28}$ It is also of note that growth factors, such as vascular endothelial growth factor (VEGF), can induce the release of vWf from endothelial cells in vitro. ${ }^{29}$

In the eye, numerous types of retinal cells are recognised to produce VEGF, including retinal pigment epithelial cells, pericytes, endothelial cells, Muller cells, and astrocytes. ${ }^{18}$ Intraocular VEGF levels have also been studied in animal models and human vitreous fluid, where the levels are found to be high in patients with active intraocular neovascularisation, such as proliferative diabetic retinopathy, ischaemic central retinal vein occlusion, rubeosis iridis, and retinopathy of prematurity. ${ }^{18}$ Changes in intraocular VEGF levels have also been related to effective laser treatment. ${ }^{18}$ We recently reported a pilot study of patients with proliferative retinopathy, who had significantly raised plasma levels of VEGF and vWf when compared to diabetics with background retinopathy only and healthy controls. ${ }^{22}$ Following panretinal laser photocoagulation of the patients with proliferative retinopathy, there was a significant reduction in plasma VEGF levels at 4 months follow up but no significant changes in plasma sFlt-l or vWf levels; importantly, patients with complete resolution had a trend towards lower median VEGF levels. ${ }^{22}$ Furthermore, additional work from our group has demonstrated persisting abnormalities in haemorheological factors and vWf in retinal vascular occlusion, ${ }^{21}$ as well as abnormal levels of plasma VEGF and haemorheological markers in patients with age related macular degeneration. ${ }^{23}$

In the present study, median levels of sFlt-1 were significantly lower in patients with NTG and POAG, when compared to healthy controls-as we previously reported in vascular disease..$^{30}$ The amount of sFlt-1 (measured as mass or mol) greatly exceeds that of VEGF, and the ELISA measures not simply the presence of sFlt- 1 , but that sFlt- 1 which binds to VEGF (that is, a pseudo-bioassay), so that our finding may have biological relevance if, as seems possible, one function of sFlt- 1 is to regulate plasma VEGF levels. ${ }^{30-32}$ Consequently, this excess of functional (VEGF binding) sFlt-1 over VEGF may frustrate the proposed use of VEGF as a potential therapeutic agent, as any exogenously administered VEGF would need to neutralise any circulating sFlt- 1 before achieving therapeutic levels at target receptors/organs. The further pathophysiological consequences of sFlt-l are unknown in glaucoma and, clearly, the present (novel) findings in this hypothesis testing pilot study will require further detailed mechanistic/ interventional studies in larger groups of patients.

In conclusion, this pilot study suggests that the pathogenesis of optic nerve damage in both NTG and POAG may be associated with abnormal vascular permeability and endothelial damage/dysfunction, as reflected by abnormal VEGF and vWf levels respectively in these patients. 


\section{ACKNOWLEDGEMENTS}

This project is supported by the Royal College of Surgeons of Edinburgh "Pump Priming Grant" (The Royal Blind Asylum and School/Scottish National Institute for the War Blinded Fund). We acknowledge the support of the City Hospital Research and Development Programme for the Haemostasis Thrombosis and Vascular Biology Unit. We thank Dr S Ramanathan and Dr F Belgore for assistance with data collection and sample processing.

\section{Authors' affiliations}

P L Lip, D C Felmeden, A D Blann, G Y H Lip, Haemostasis, Thrombosis and Vascular Biology Unit, University Department of Medicine, City Hospital, Birmingham, UK

P L Lip, N Matheou, S Thakur, I A Cunliffe Birmingham and Midland Eye Centre, City Hospital, Birmingham, UK

\section{REFERENCES}

1 Carter CJ, Brooks DE, Doyle DL, Drance SM. Investigations into a vascular aetiology for low-tension glaucoma. Ophthalmology 1989;97:49-55.

2 Sonnsio B, Krakau CE. Arguments for a vascular glaucoma aetiology Acta Ophthalmol 1993;71:433-4.

3 Henry E, Newby EN, Webb DJ, et al. Peripheral endothelial dysFunction in normal pressure glaucoma. Invest Ophthalmol Vis Sci.1999;40:17104

4 Armaly MF. Lessons to be learned from the Collaborative Glaucoma Study. Surv Ophthalmol 1981;99:837-9.

5 Leske MC, Podgor MC. Intraocular pressure, cardiovascular risk variables and visual field defects. Am J Epidemiol 1983;1 18:280-7.

6 Jampol LM, Board RJ, Maumence AE. Systemic hypotension and glaucomatous changes. Am J Ophthalmol 1978;85:154-9.

7 Drance SM, Sweeney VP, Organ RW, et al. Studies of factors involved in the production of low-tension glaucoma. Arch Ophthalmol 1973;89:457-65

8 Winder AF. Circulatory lipoprotein and blood glucose levels in association with low tension and chronic simple glaucoma. $\mathrm{Br}$ Ophthalmol 1977:61:642-5.

9 Joist JH, Linchtenfeld P, Mandell Al, et al. Platelet function, blood coagulability, and fibrinolysis in patients with low-tension glaucoma. Arch Ophthalmol 1976;94:1893-5.

10 Klaver JHJ, Greve EL, Goslinga H, et al. Blood and plasma viscosity measurements in patients with glaucoma.

11 O'Brien C, Butt Z, Ludlam C, et al. Activation of the coagulation cascade in untreated primary open-angle glaucoma. Ophthalmology 1997; 104:725-30

12 Tsakiris DA, Osusky R, Kaiser HJ, et al. Lupus anti-coagulants/ anticardiolipin antibodies in patients with normal tension glaucoma. Blood Coagl Fibrinolysis 1992;3:541-5.

13 Levene Rz. Low tension glaucoma: a critical review and new material Surv Ophthalmol 1980;24:139-64.

14 Dvorak HF, Brown LF, Detmat $M$, et al. Vascular permeability factor/vascular endothelial growth factor, microvascular hypermeability, and angiogenesis. Am J Pathol 1995;146:1029-39.
15 Leung DW, Cachianes G, Kuang WJ, et al. Vascular endothelial growth factor is a secreted angiogenic mitogen. Science 1989;246:1306-9.

16 Kondo S, Asano M, Matsuo K, et al. Vascular endothelial growth factor/vascular permeability factor is detectable in the serum of tumour bearing mice and in cancer patients. Biochim Biophys Acta 1994; 1221:211-14.

17 Miller JW, Adamis AP, Shima DT, et al. Vascular endothelial growth factor/vascular permeability factor is temporally and spatially correlated with ocular angiogenesis in a primate model. Am J Pathol 1994; 145:574-84.

18 Aeillo LP, Avery RL, Arigg PG, et al. Vascular endothelial growth factor in ocular fluids of patients with diabetic retinopathy and other retinal disorders. N EnglJ Med 1994;331:1480-7

19 Barleon B, Hauser S, Schollmann C, et al. Differential expression of the two VEGF receptors Flt-1 and KDR in placenta and vascular endothelial cells. J Cell Biochem 1994;54:56-66.

20 Kendall RL. Wang G. Thomas KA. Identification of a natural soluble form of the vascular endothelial growth factor receptor, FLT-1, and its heterodimerization with KDR. Biochem Biophys Res Commun 1996;226:324-8

21 Lip PL, Blann AD, Jones AF, et al. Persisting abnormalities in haemorheological factors and lipoprotein(a) in retinal vascular occlusion: Implications for increased vascular risk. Eye 1998;12:245-51.

22 Lip PL, Belgore F, Blann AD, et al. Plasma VEGF and soluble VEGF receptor Flt-1 in proliferative retinopathy: relationship to endothelial dysfunction and laser treatment. Invest Ophthalmol Vis Sci 2000:41:2115-19.

23 Lip PL, Blann AD, Hope-Ross MW, et al. Age-related macular degeneration is associated with increased vascular endothelial growth factor, haemorheology and endothelial dysfunction. Ophthalmology $2001 ; 108: 705-10$

24 Peduzzi M, Melli M, Fonda S, et al. Comparative evaluation of blood viscosity in daibetes retinopathy. Int Ophthalmol 1984;7:15-9.

25 Ring CP, Pearson TC, Sanders MD, et al. Viscosity and retinal vein thrombosis: Br J Ophthalmol 1976;60:397-410.

26 Lip GYH, Blann AD. von Willebrand factor and its relevance to cardiovascular disorders. Br Heart J 1995;74:580-3.

27 Blann AD. Increased release of von Willebrand factor antigen by endothelial cells whilst in active growth phase. Cell Biol Int Reps $1991 ; 15: 493-8$

28 Olsson A, Elling P, Elling $\mathrm{H}$. Serological and immunohistological determination of $\mathrm{vWf}$ antigen in serum and biopsy specimens from patients with arteriris temporalis and polymyalgia rheumatica. Clin Exp Rheum 1990;8:55-8.

29 Brock TA, Dvorak HF, Senger DR. Tumour-secreted vasular permeability factor increases $\mathrm{Ca}$ and von Willebrand factor release in human endothelial cells. Am J Path 1991;138:213-21.

30 Blann AD, Belgore FM, McCollum CN, et al. Vascular endothelial growth factor and its receptor, Flt-1, in the plasma of patients with coronary or peripheral atherosclerosis, or Type II diabetes. Clin Sci (Lond) 2002;102:187-94.

31 Kendall RL, Thomas KA. Inhibition of vascular endothelial growth factor activity by an endogenously encoded soluble receptor. Proc Natl Acad Sci USA 1993;90: 10705-9.

32 Goldman CK, Kendall RL, Cabbera G, et al. Paracrine expression of a native soluble vascular endothelial growth factor receptor inhibits tumour growth, metastasis and mortality rate. Proc Natl Acad Sci USA 1998;95:8795-800. 